\title{
The Impact of a 1950s Era Metallurgy Article on the Fields of Mathematics and Biology
}

(C) Springer Science+Business Media New York and ASM International 2015

\section{Editor's Note}

The following article, "Grain Shapes and Other Metallurgical Applications of Topology" by Cyril Stanley Smith, was brought to our attention by William Waites, a researcher at the University of Edinburgh's School of Informatics. Like many of us, he participates in a journal club, wherein a publication of interest is presented and discussed with colleagues. This particular journal club focuses on modeling of biological systems, so it came as a bit of a surprise that there was interest in a paper by Cyril Stanley Smith that published in Metal Interfaces in 1952 (65-113, American Society for Metals, Cleveland, OH).

The relationship to biology is revealed in the discussion section at the end of the paper. Dr. John von Neumann, of the Institute for Advanced Study, Princeton University, discusses what has become known as the von Neumann Law, or the von Neumann-Mullins relationship, which describes the rate of change of area of a bubble. This relationship has wide-reaching implications into many fields, including biology where it can describe the changes that occur in cells. It is interesting, and not altogether surprising, that a paper on grain shapes from a prominent metallurgist would generate influential discussion relating to the field of biology. Indeed, another discussion author, Columbia University botany professor Edwin Matzke, observed that Smith's paper "serves to bring into focus the close interrelationship, within limits, of concepts of space partition in such diverse fields as mathematics, metallurgy, and cellular configurations in plant and animal tissues." This is a reminder to all of us that our work may have impact beyond the immediate application for which it was intended.

Smith's original manuscript is not widely available, despite the impact it has had across several fields of study. It is reproduced here in the hopes that researchers and scientists who utilize and refer to von Neumann's Law in their work will benefit from reading the manuscript that generated this important discussion. I extend our gratitude to William Waites for bringing this paper to our attention, and invite our readers to suggest other influential, yet not widely available, manuscripts that should be considered for reproduction here. Comments and suggestions can be sent to ryan.deacon@asminternational.org. 